\title{
Prevalencia y enfoque terapéutico del dolor en el servicio de urgencias de un hospital universitario
}

\author{
S. Mínguez Masó1, R. Herms Puig², E. Arbonés $\operatorname{Aran}^{2}$, C. Roqueta Guillén ${ }^{3}$, C. Farriols Danés ${ }^{4}$, \\ M. Riu Camps y A. Montes Pérez ${ }^{5}$
}

Servicios de ${ }^{1}$ Urgencias y ${ }^{2}$ Anestesiología, Reanimación y Terapéutica del Dolor. Hospital del Mar. Parc de Salut Mar. Barcelona. ${ }^{3}$ Servicio de Geriatría. Centro Fórum-Hospital del Mar. Parc de Salut Mar. Barcelona. ${ }^{4}$ Unidad de Cuidados Paliativos. Servicio de Oncología Médica. Hospital de la Esperanza. Parc de Salut Mar. Barcelona. ${ }^{5}$ Servicio de Epidemiología y Evaluación. Parc de Salut Mar. Barcelona. Grupo de Epidemiología y Evaluación IMIM (Hospital del Mar Medical Research Institute). Barcelona. Comisión para la Evaluación y Tratamiento del Dolor. Parc de Salut Mar. Hospital del Mar. Barcelona

Mínguez Masó S, Herms Puig R, Arbonés Aran E, Roqueta Guillén C, Farriols Danés C, Riu Camps M, Montes Pérez A. Prevalencia y enfoque terapéutico del dolor en el servicio de urgencias de un hospital universitario. Rev Soc Esp Dolor 2014; 21(4): 205-211.

\begin{abstract}
Aim: To know the prevalence of pain and the therapeutic approach in an emergency department (ED).

Material and methods: Cross-sectional study in the ED of a university hospital during 7 consecutive days was performed. The presence of pain was asked by a physician to patients older than 15 years admitted at ED. Patients admitted at Psychiatry, Obstetrics-Gynecology Emergency Department and those whose serious condition would not allow pain assessment in triage were excluded. The previous analgesic treatment, pain assessment, analgesic treatment in the ED and also scheduled treatment at discharge were later reviewed.

Results: Six hundred and sixty-eight from 2,287 patients who were admitted at ED were analyzed. Five hundred and one patients reported pain (75\%), of which 45 were admitted and 456 were discharged at home (240 patients in level 1 , 216 at level 2). The $24.5 \%$ of the 501 patients who reported pain at admission were previously treated with analgesics. The $41.7 \%$ of the 216 patients treated at level 2 received analgesic treatment during their stay [paracetamol (80.2\%), nonsteroidal antiinflammatory drugs (NSAIDs) (50.0\%), co-analgesics $(1.0 \%)$, weak opioids (20.8\%), strong opioids (3.1\%)]. The $32.7 \%$ of the 196 patients without documented pain assessment received analgesic treatment as required. In 69.4 $\%$ of the 456 patients who did not require hospitalization, analgesic treatment was prescribed or modified at discharge [paracetamol (55\%), nonsteroidal anti-inflammatory drugs
\end{abstract}

Recibido: 15-07-13.

Aceptado: 20-12-13.
(NSAIDs) (67\%), co-analgesics (10\%), weak opioids (10\%), strong opioids (1.1\%)].

Conclusions: A high prevalence of pain (75\%) was found in patients admitted at ED. The $41.7 \%$ received analgesic treatment during their stay and in $69.4 \%$ of patients analgesia was prescribed at discharge. Low opioid prescription was detected.

Key words: Pain. Analgesics. Emergency department. Hospital. Triage.

\section{RESUMEN}

Objetivo: conocer la prevalencia de dolor y su enfoque terapéutico en un servicio de urgencias.

Material y métodos: estudio transversal llevado a cabo en el Servicio de Urgencias de un hospital universitario durante 7 días consecutivos. El médico de triaje interrogó sobre la presencia de dolor a los pacientes mayores de 15 años que acudían a urgencias. Se excluyeron los pacientes atendidos en el Servicio de Psiquiatría, Obstetricia-Ginecología y aquellos cuya gravedad no permitía la valoración del dolor en el triaje. Se revisó posteriormente el tratamiento analgésico previo a la visita, la evaluación del dolor, el tratamiento analgésico aplicado durante su estancia en urgencias y el tratamiento pautado al alta.

Resultados: se analizaron 668 de los 2.287 pacientes que acudieron a urgencias durante el periodo del estudio. Presentaron dolor 501 pacientes (75\%), de los que 45 ingresaron y 456 fueron dados de alta a domicilio (240 pacientes procedentes de nivel 1 y 216 de nivel 2). El 24,5\% de los 501 pacientes que manifestaron tener dolor realizaba tratamiento analgésico previo. De los 216 pacientes atendidos en el nivel 2, el 41,7\% recibió tratamiento analgésico durante su estancia [paracetamol (80,2 \%), antiinflamatorios no esteroideos (AINE) (50\%), coanalgésicos (1\%), opioides débiles (20,8\%), opioides fuertes (3,1 \%)]. El 32,7 \% de los 196 pacientes sin registro de evaluación del dolor recibió tratamiento analgésico a demanda. En un $69,4 \%$ de los 456 pacientes que no precisaron ingreso, al alta se pautó analgesia o se modificó el tratamiento analgésico pre- 
vio [paracetamol (55\%), AINE (67\%), co-analgésicos (10\%), opioides débiles (10\%), opioides fuertes (1,1\%)].

Conclusiones: se evidencia una elevada prevalencia de dolor (75\%) en los pacientes que acuden al servicio de urgencias. El $41,7 \%$ de los pacientes que presentaban dolor recibió tratamiento analgésico durante su estancia y en el 69,4\% de los pacientes que no precisaron ingreso se pautó analgesia al alta. Destaca la baja prescripción de opioides.

Palabras clave: Dolor. Analgésicos. Servicio de urgencias. Hospital. Triaje.

\section{INTRODUCCIÓN}

El dolor es uno de los motivos más frecuentes de consulta en los servicios de urgencias (1), ya sea directamente o por patologías que lo ocasionan. La prevalencia de dolor en los pacientes de urgencias es muy alta, alrededor del $78 \%(2,3)$ pero, a pesar de este alto porcentaje, numerosos estudios han demostrado que el manejo de este síntoma es inadecuado en muchas ocasiones $(4,5)$. Las causas son diversas: los escasos conocimientos de los profesionales (4), la dificultad para aplicar un sistema de valoración del dolor eficaz o la mayor preocupación por el diagnóstico que por el tratamiento (3).

El dolor como síntoma genera gran sensación de urgencia $(6,7)$ y puede ocasionar un aumento de la gravedad del paciente por los cambios hemodinámicos y neurohormonales que puede llegar a desencadenar (8). Los pacientes suelen recibir tratamiento analgésico subóptimo, ya sea por pautas inadecuadas, dosis insuficientes o fármacos inapropiados. Este fenómeno, conocido como "oligoanalgesia", ocurre en un alto porcentaje de pacientes (3) y no únicamente en los servicios de urgencias (9).

Para el adecuado tratamiento del dolor es primordial su evaluación tanto en el triaje (en los sistemas de triaje la presencia de dolor hace que se priorice la necesidad de asistencia) como durante la estancia en urgencias $(1,8,10)$. La Joint Comission on Accreditation of Healthcare Organizations (11) estableció la evaluación del dolor como la quinta constante debido a la baja frecuencia de su valoración.

Ante esta situación y con motivo de que el año 2011 fue declarado por la International Association for the Study of Pain (IASP) como "Año Global contra el Dolor Agudo", realizamos un estudio en nuestro centro para conocer la prevalencia de dolor en urgencias y su abordaje terapéutico.

\section{MATERIAL Y MÉTODOS}

Estudio transversal, llevado a cabo en el servicio de Urgencias de un Hospital Universitario de Barcelona, del 6-12 de junio del año 2011, durante las 24 horas del día.
La población estaba formada por todos los pacientes mayores de 15 años que acudieron al servicio de Urgencias durante el periodo de estudio. Se excluyeron los pacientes atendidos en el Servicio de Psiquiatría, Obstetricia-Ginecología y aquellos cuya gravedad no permitía la valoración del dolor en el triaje.

El estudio se llevó a cabo en un Servicio de Urgencias jerarquizado con una media de 87.000 asistencias anuales. En este servicio el triaje se realiza por un médico adjunto que interroga a todos los pacientes (excepto los pertenecientes al área de pediatría) y registra en un sistema informático el motivo de consulta y la especialidad que visitará al paciente (medicina, traumatología u otras especialidades quirúrgicas). También determina la ubicación del paciente según su gravedad (nivel 1 en los casos de baja complejidad diagnóstica y terapéutica o nivel 2 en pacientes de mayor gravedad).

Para el estudio se creó una casilla en la pantalla de triaje donde hacer constar si el paciente tenía o no dolor. El médico responsable preguntaba a todos los pacientes, independientemente del motivo de la consulta, si tenían algún tipo de dolor.

Posteriormente se revisó la historia clínica de todos los pacientes que manifestaron presentar algún tipo de dolor, con el fin de analizar el tratamiento analgésico previo. De los pacientes ubicados en nivel 2 y que no precisaron ingreso hospitalario, se analizó la valoración del dolor y el tratamiento analgésico aplicado durante su estancia en urgencias.

En todos los pacientes dados de alta (nivel 1 y nivel 2) se evaluó el tratamiento analgésico pautado en el informe de alta.

El resto de variables analizadas fueron: edad, sexo, motivo de la consulta (codificado según la CIE-9-MC), especialidad médica que atendió la urgencia y si el paciente fue dado de alta desde urgencias o precisó ingreso hospitalario. Este estudio no supuso ninguna modificación en los protocolos habituales de asistencia y los datos se trataron de forma anónima.

Se realizó un análisis descriptivo de los datos, de forma global y según la especialidad que atendió la urgencia. La edad se categorizó según los cuartiles de la distribución. Para la comparación de las variables se utilizó el test de Chi cuadrado o el test exacto de Fisher si un valor esperado era inferior a cinco.

\section{RESULTADOS}

La distribución de pacientes se muestra en el diagrama de flujo del gráfico que se muestra en la figura 1. De los 2.287 pacientes que acudieron a urgencias en el periodo analizado 1.138 cumplían los criterios de inclusión. La valoración del dolor se realizó en 668 de ellos $(58,7 \%)$. En la tabla I se 


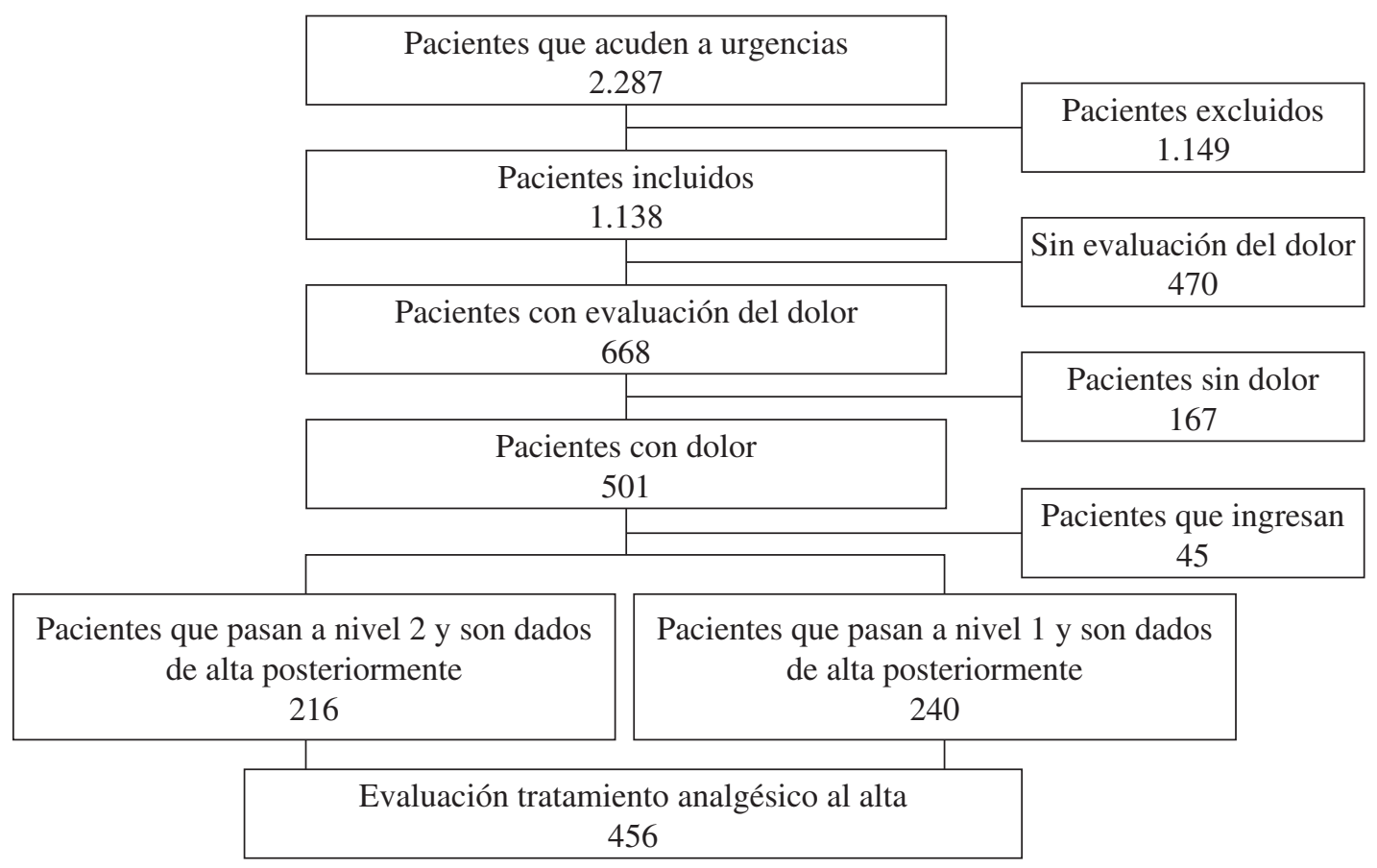

Fig. 1. Diagrama de flujo de pacientes.

TABLA I. DISTRIBUCIÓN DE LOS PACIENTES EN GRANDES GRUPOS DIAGNÓSTICOS DE LA CIE-9-MC

\begin{tabular}{|c|c|c|c|c|}
\hline \multirow[t]{2}{*}{ Grandes grupos diagnósticos CIE-9-MC } & \multirow[t]{2}{*}{ Total pacientes } & \multicolumn{2}{|c|}{ Pacientes incluidos } & \multirow[t]{2}{*}{$p$} \\
\hline & & $n$ & $\%$ & \\
\hline E. infecciosas y parasitarias & 26 & 18 & 69,2 & \\
\hline Neoplasias & 16 & 9 & 56,3 & \\
\hline E. endocrinas, nutric., metabol. y trast. inmunidad & 6 & 4 & 66,7 & \\
\hline E. sangre y órganos hematopoyéticos & 9 & 3 & 33,3 & \\
\hline Trastornos mentales & 29 & 15 & 51,7 & \\
\hline E. sistema nervioso y órganos sentidos & 40 & 27 & 67,5 & \\
\hline E. aparato circulatorio & 75 & 41 & 54,7 & \\
\hline E. aparato respiratorio & 85 & 53 & 62,4 & \\
\hline E. aparato digestivo & 103 & 62 & 60,2 & \\
\hline E. aparato genitourinario & 59 & 41 & 69,5 & \\
\hline E. piel y tejido subcutáneo & 57 & 37 & 64,9 & \\
\hline E. aparato locomotor y tejido conectivo & 117 & 79 & 67,5 & \\
\hline Lesiones y envenenamientos & 154 & 92 & 59,7 & \\
\hline Síntomas, signos y afecciones mal definidas & 315 & 164 & 52,1 & \\
\hline Factores que influyen en el estado de salud & 47 & 23 & 48,9 & \\
\hline Total & 1.138 & 668 & 58,7 & 0,082 \\
\hline
\end{tabular}


muestran los pacientes agrupados según grandes grupos de diagnóstico de la CIE-9-MC, no se observaron diferencias estadísticamente significativas entre los pacientes a los que se valoró el dolor y a los que no $(\mathrm{p}=0,082)$.

La prevalencia global del dolor fue del $75 \%$ (Tabla II). $\mathrm{El}$ grupo de enfermedades del aparato locomotor y tejido conectivo, fue el que tuvo una mayor prevalencia $(93,7 \%)$.

De los 668 pacientes a los que se evaluó el dolor, 343 eran hombres $(51,3 \%)$ y 325 mujeres $(48,7 \%)$. La media de edad global fue de 50,6 $\pm 21,95$ años. El 51,6\% (345) fue atendido en el área de medicina, 27,4 \% (183) en traumatología y $21 \%$ (140) en otras especialidades quirúrgicas.

En la tabla III se muestra la prevalencia de dolor global y por especialidades, y también según sexo, grupo de edad y destino al alta de urgencias. El $80 \%$ de las mujeres presentaron dolor, así como el $70 \%$ de los hombres ( $\mathrm{p}=0,004)$. Los pacientes del área de traumatología presentaron una mayor prevalencia de dolor $(94,0 \%)$ con una diferencia estadísticamente significativa $(\mathrm{p}<0,001)$ respecto a los de las áreas restantes.

De los 501 pacientes que manifestaron tener dolor a su llegada a urgencias, el 24,5\% realizaba algún tipo de tratamiento analgésico previo: paracetamol (37,9\%), AINE (40\%), co-analgésicos $(8,1 \%)$, opioides débiles $(9,9 \%)$ y opioides fuertes $(4,1 \%)$.
Durante la estancia en urgencias recibieron tratamiento analgésico el 41,6\% de los 216 pacientes con dolor atendidos en el nivel 2 y que no precisaron ingreso hospitalario (Tabla IV). El registro de la evaluación del dolor de los 216 pacientes, se realizó en 20 pacientes (13,2\%). De los 196 pacientes en los que no se registró la evaluación del dolor, 64 pacientes $(32,6 \%)$ recibieron tratamiento analgésico pautado a demanda. El tratamiento analgésico realizado se muestra en la tabla IV.

El análisis del tratamiento analgésico pautado en el informe de alta en los 456 pacientes no tributarios de ingreso hospitalario ( $84,5 \%$ de los casos), mostró que en un $69,4 \%$ se pautó analgesia o se modificó el tratamiento analgésico previo [paracetamol (55\%), AINE (67\%), co-analgésicos (10\%), opioides débiles (10\%), opioides fuertes $(1,1 \%)]$.

\section{DISCUSIÓN}

El estudio refleja la alta prevalencia del dolor en el servicio de urgencias, hecho que corrobora que el dolor es uno de los principales motivos de consulta en esta área. La prevalencia global del dolor en nuestro estudio fue de $75 \%$, similar a la reportada por otros grupos (3). El dolor

TABLA II. PREVALENCIA DE DOLOR EN LOS PACIENTES, DISTRIBUCIÓN POR GRANDES GRUPOS DIAGNÓSTICOS DE LA CIE-9-MC

\begin{tabular}{lcc}
\hline \multicolumn{1}{c}{ Grandes grupos diagnósticos CIE-9-MC } & Pacientes con dolor & Prevalencia dolor, \% \\
\hline E. infecciosas y parasitarias & 9 & 50,0 \\
Neoplasias & 7 & 77,8 \\
E. endocrinas, nutric., metabol. y trast. inmunidad & 2 & 50,0 \\
E. sangre y órganos hematopoyéticos & 1 & 33,3 \\
Trastornos mentales & 7 & 46,7 \\
E. sistema nervioso y órganos sentidos & 18 & 66,7 \\
E. aparato circulatorio & 25 & 61,0 \\
E. aparato respiratorio & 28 & 52,8 \\
E. aparato digestivo & 49 & 79,0 \\
E. aparato genitourinario & 32 & 78,0 \\
E. piel y tejido subcutáneo & 22 & 59,5 \\
E. aparato locomotor y tejido conectivo & 74 & 93,7 \\
Lesiones y envenenamientos & 72 & 78,3 \\
Síntomas, signos y afecciones mal definidas & 140 & 85,4 \\
Factores que influyen en el estado de salud & 15 & 65,2 \\
\hline Total & 501 & 75,0 \\
\hline
\end{tabular}



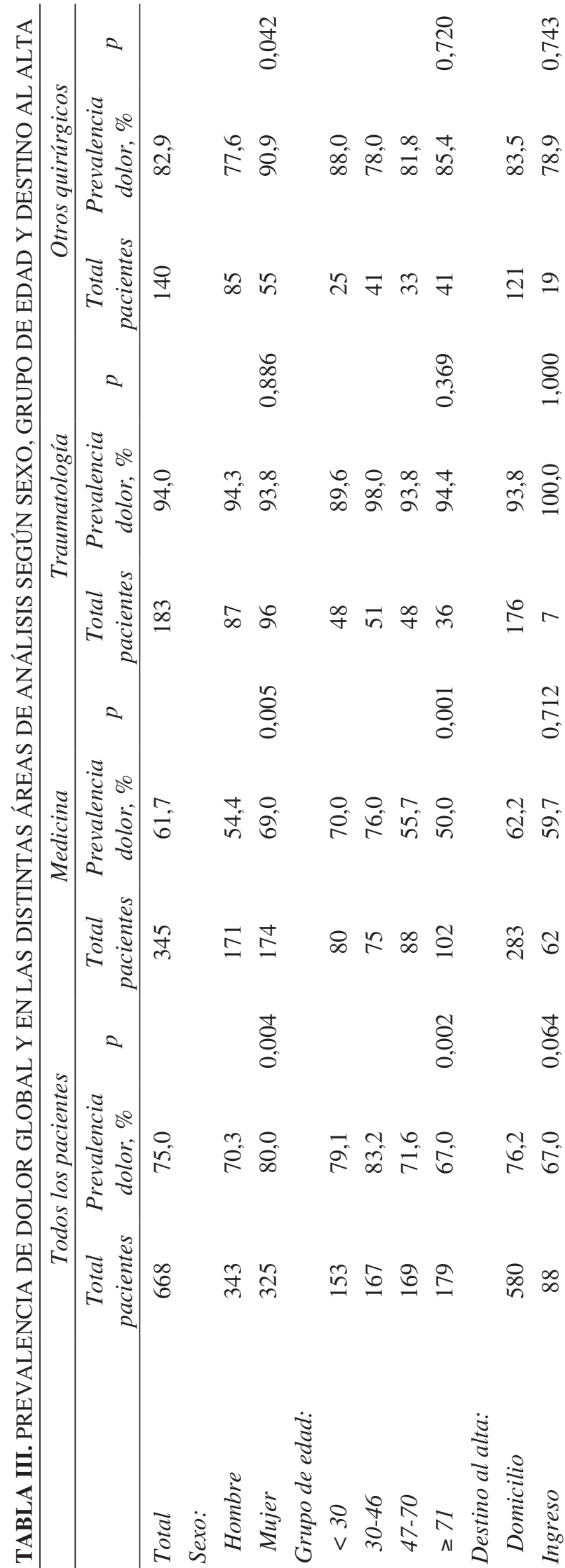

\section{EN LOS PACIENTES DEL NIVEL 2}

TABLA IV.TRATAMIENTO ANALGÉSICO REALIZADO

\begin{tabular}{lll}
\hline & \multicolumn{2}{c}{$\begin{array}{c}\text { Número de } \\
\text { pacientes }\end{array}$} \\
& $\mathrm{n}$ & $\%$ \\
\hline Total pacientes & 216 & -- \\
Pacientes con tratamiento analgésico & 96 & 41,7 \\
Tipo de analgésico pautado: & & \\
Paracetamol & 77 & 80,2 \\
AINE & 48 & 50,0 \\
Co-analgésicos & 1 & 1,0 \\
Opioides débiles & 20 & 20,8 \\
Opioides fuertes & 3 & 3,1 \\
\hline
\end{tabular}


el mismo autor en cinco países de la Comunidad Europea $(0,34$ vs. 0,$18 ; \mathrm{p}<0,05)(17)$.

La valoración y el registro del dolor en el área de urgencias sigue siendo deficiente a pesar de los múltiples estudios publicados sobre el beneficio del uso de escalas (18). El escepticismo de los médicos y la prioridad en el diagnóstico inmediato son factores que contribuyen a esta infravaloración (19). En este estudio, el registro de la evaluación del dolor en los 216 pacientes atendidos en el nivel 2 y que no precisaron ingreso hospitalario, se realizó sólo en un $13,2 \%$ de los casos. En los pacientes en que no se registró la evaluación del dolor, un 32,6 \% recibió el tratamiento analgésico pautado a demanda, por lo que se puede inferir que en realidad sí se realizó una valoración del dolor aunque no se registró. Este hecho confirma el dato ya conocido de la insuficiente evaluación y registro mediante escalas validadas del dolor en urgencias, así como la falta de protocolos o algoritmos consensuados. Nelson y cols. (20) observaron que la utilización de escalas de valoración del dolor en urgencias, aumentó el uso de analgesia de un $25 \%$ a un $36 \%$ y que dicho tratamiento fue administrado más rápidamente. Baumann y cols. (21) mostraron que evaluar el dolor mediante una escala y registrarlo en la documentación clínica aumentaba la proporción de pacientes en los que se valoraba el dolor.

En nuestro estudio el 58,3\% de los pacientes atendidos en el nivel 2, no recibieron tratamiento analgésico a pesar de referir dolor, aunque sí se realizó una prescripción farmacológica al alta. Una cifra muy elevada dada la importancia del tratamiento del dolor en el área de urgencias. Son diversos los estudios publicados sobre el fenómeno de la oligoanalgesia en las áreas de urgencias. Wilson y Pendleton (22) observaron que un $56 \%$ de pacientes no recibieron tratamiento analgésico durante su espera en el área de urgencias, $69 \%$ esperaron más de una hora antes de recibir analgesia y $42 \%$ más de dos horas. Además, de los pacientes que recibieron analgesia, el $32 \%$ recibió menor dosis de la considerada óptima. En otros estudios $(23,24)$, únicamente recibieron analgesia entre un $30 \%$ y un $49 \%$ de los pacientes que acudieron a urgencias por fractura ósea aguda.

Los analgésicos mayoritariamente pautados, tanto durante su permanencia en el área como al alta, fueron paracetamol y AINE, siendo baja la prescripción de opioides tanto débiles como fuertes. Todd y cols. (3), en un estudio multicéntrico realizado en 20 servicios de urgencias analizaron la prescripción de analgésicos durante la estancia en urgencias y objetivaron, a diferencia de nuestro estudio, que la administración de opioides fue más elevada (59\% de los analgési$\cos )$, siendo el de uso más frecuente la morfina (20\%). Este hecho se refleja en otros estudios $(9,25)$.

Cabe destacar el potencial de intervención farmacológica del Servicio de Urgencias. En el presente estudio se objetivó un elevado número de prescripciones (pacientes sin tratamiento previo prescrito) y de modificaciones de tratamiento a pesar que globalmente el dolor fue infratratado. El porcentaje de prescripción de analgésicos al alta $(69,41 \%)$, durante la semana del estudio fue elevada, aunque debería mejorarse. Por lo tanto, debemos tomar conciencia de la importancia del abordaje terapéutico del dolor en urgencias dado que es un problema sanitario de gran relevancia y prevalencia.

A pesar que se ha descrito en la literatura un fenómeno creciente de abuso de opioides (2), en nuestro estudio llama la atención el bajo porcentaje de prescripción de opioides potentes $(3,1 \%$ en pacientes ingresados en el servicio de urgencias y $1,1 \%$ al alta). Este hecho refleja el fenómeno de opiofobia, que se define como prejuicio en contra del uso y prescripción de analgésicos opioides. Algunos motivos podrían ser la falta de conocimiento sobre opioides, el miedo a enmascarar síntomas de la enfermedad aguda, la dificultad burocrática en la prescripción, el respeto a los efectos secundarios, la preocupación por el abuso o dependencia y la dificultad en el seguimiento (19). El resultado de la opiofobia es que los pacientes no reciben el tratamiento analgésico apropiado o lo reciben a dosis inadecuadas, y en muchas ocasiones son dados de alta del servicio de urgencias con dolor y sin la prescripción de un analgésico opioide adecuado (19).

En cuanto a las limitaciones del presente estudio destacamos la ausencia de instrumentos de medida para la valoración de la intensidad y de las características del dolor, a diferencia de otros estudios publicados en la literatura médica $(19,26)$. Aunque el objetivo inicial del estudio fue establecer la prevalencia del dolor, el conocer su intensidad nos hubiera proporcionado más datos sobre la adecuación del tratamiento. A diferencia de otros estudios, no fueron evaluados el tiempo de demora hasta el inicio del tratamiento analgésico ni la respuesta al tratamiento farmacológico instaurado. A pesar del elevado número de pacientes no valorados durante el periodo de reclutamiento, cabe destacar que la muestra de los pacientes no evaluados ha sido homogénea con respecto a los valorados.

Una revisión de los diferentes protocolos de tratamiento del dolor en los servicios de urgencias de Holanda, mostró diferencias considerables en el enfoque terapéutico ante una misma situación clínica (27). El manejo terapéutico del dolor agudo debería ser interdisciplinar, intensivo desde el inicio, con un uso adecuado de opioides y valorando asimismo medidas no farmacológicas (27). Es importante que los algoritmos sean simples y favorezcan la toma adecuada de decisiones (5). Además, es fundamental la formación y el conocimiento de los médicos sobre aspectos de valoración y tratamiento del dolor agudo (28). En nuestro centro, la comisión de evaluación y tratamiento del dolor tiene entre sus funciones la de establecer protocolos multidisciplinares adecuados a las necesidades de las distintas áreas, incluido el servicio de urgencias. 
Un correcto seguimiento por los profesionales de Atención Primaria contribuye a un mejor cumplimiento terapéutico y favorece el control de la efectividad y la tolerancia. En este sentido, la informatización de la historia clínica permite una historia compartida con Atención Primaria facilitando la continuidad asistencial $(2,5)$. Fomentar la investigación con estudios prospectivos podría mejorar la evidencia científica y la calidad asistencial de los servicios de urgencias.

\section{AGRADECIMIENTOS}

Los autores agradecen la aportación de los investigadores colaboradores con la recogida de datos de los pacientes, sin los cuales no se hubiera podido llevar a cabo el estudio.

Investigadores colaboradores: Alfonso Aguirre Tejedo, María Dolores Aranda Cárdenas, María Luisa Blanco López, Isabel Campodarve Botet, Luis Cuixart Costa, José Luis Echarte Pazos, Alejandro Miguel Galán Aisa, M. ${ }^{2}$ Teresa Martínez Izquierdo, María Dolores Marzo Roche, Jorge Mario Mejía Kattah, Oriol Pallás Villaronga, Lucilla Piccari, Margarita Puiggalí Ballart, María Dolores Carmen Sánchez Rodríguez, Augusto Supervía Caparrós, María Pilar Villafranca Tejero.

\section{CORRESPONDENCIA}

Silvia Mínguez Masó

Servicio de Urgencias

Hospital del Mar

Parc de Salut Mar

Passeig Marítim de la Barceloneta, 25-29

08003 Barcelona

e-mail: SMinguez@parcdesalutmar.cat

\section{BIBLIOGRAFÍA}

1. Vazirani J, Knott J. Mandatory pain scoring at triage reduces time to analgesia. Ann Emerg Med 2012;59:134-8.

2. Todd KH. Pain and prescription monitoring programs in the emergency department. Ann Emerg Med 2010;56:24-6.

3. Todd KH, Ducharme J, Choiniere M, et al. Pain in the emergency department: Results of the Pain and Emergency Medicine Initiative (PEMI) multicenter study. J Pain 2007;8:460-6.

4. Gordon DB, Dahl JL, Miaskoswki C, et al. American Pain Society Recommendations for improving the Quality of Acute and Cancer Pain Management: American Pain Society Quality of Care Task Force. Arch Intern Med 2005; 165:1574-80.

5. Tamchès E, Buclin T, Hugli $\mathrm{O}$, et al. Acute pain in adults admitted to the emergency room: Development and implementation of abbreviated guidelines. Swiss Med Wkly 2007;137:223-7.

6. Carbonell Torregrosa MA, Girbés Borrás J, Calduch Broseta JV. Determinantes del tiempo de espera en urgencias hospitalaria y su relación con la satisfacción del usuario. Emergencias 2006;18:30-5.

7. Pasarín MI, Fernández de Sanmamed MJ, Calafell J, et al. Razones para acudir a los servicios de urgencias hospitalarios. La población opina. Gac Sanit 2006;20:91-9.

8. Casal Codesido JR, Vázquez Lima MJ. Abordaje del dolor musculoesquelético en urgencias. Emergencias 2012;24:59-65.

9. Todd KH. Emergency medicine and pain: A topography of influence. Ann Emerg Med 2004;43:504-6.

10. Gómez-Jiménez J, Becerra O, Boneu F, et al. Análisis de la casuística de los pacientes derivables desde urgencias a atención primaria. Gac Sanit 2006;20:40-6.

11. Joint Commission on Accreditation of Healthcare Organizations. Pain assessment and management standards-Hospitals. Comprehensive Accreditation Manual for Hospitals. The official Handbook (CAMH). Oakbrook Terrace, IL: Joint Commission on accreditation of Healthcare Organizations; 2001.

12. Breivik H, Collett B, Ventafridda V, et al. Survey of chronic pain in Europe: Prevalence, impact on daily life, and treatment. Eur J Pain 2006;10:287-333.

13. http://www.doctordigital.com.ar/main/noticias-medicas/ destacadas/191-el-40-de-las-areas-de-urgencias-no-mideel-dolor-de-los-pacientes (consultado 25-07-2012)

14. Raftery KA, Smith-Coggins R, Chen AH. Gender-associated differences in emergency department pain management. Ann Emerg Med 1995;26:414-21.

15. Todd KH, Deaton C, D'Adamo AP, et al. Ethnicity and analgesic practice. Ann Emerg Med 2000;35:11-6.

16. Langley P, Pérez Hernández C, Margarit Ferri C, et al. Pain, health related quality of life and healthcare resource utilization in Spain. J Med Econ 2011;14:628-38.

17. Langley P, Müller-Schwefe G, Nicolau A, et al. The societal impact of pain in the European Union: Health-related quality of life and healthcare resource utilization. J Med Econ 2010;13:571-81.

18. Eder SC, Sloan EP, Todd K. Documentation of ED patient pain by nurses and physicians. Am J Emerg Med 2003;21:253-7.

19. Motov SM, Kan AN. Problems and barriers of pain management in the emergency department: Are we ever going to get better? Journal of Pain Research 2009;2:5-11.

20. Nelson BP, Cohen D, Lander O, et al. Mandated pain scales improve frequency of ED analgesic administration. Am J Emerg Med 2004;22:582-5.

21. Baumann BM, Colmes JH, Chansky ME, et al. Pain assessments and the provision of analgesia: the effects of a templated chart. Acd Emerg Med 2007;47-52.

22. Wilson JE, Pendleton JM. Oligoanalgesia in the emergency department. Am J Emerg Med 1989;7:620-3.

23. Lewis LM, Lasater LC, Brooks CB. Are emergency physicians too stingy with analgesics? South Med J 1994;87:7-9.

24. O'Donnell J, Ferguson LP, Beattie TF. Use of analgesia in a paediatric accident and emergency department following limb trauma. Eur J Emerg Med 2002;9:5-8.

25. Cordell WH, Keene KK, Giles BK, et al. The high prevalence of pain in emergency medical care. Am J Emer Med 2002;20:165-9.

26. Todd K. Chronic pain and aberrant drug-related behavior in the emergency department. Journal of Law, Medicine \& Ethics 2005:761-9.

27. Gaakeer MI, van Lieshout JM, Bierens JJ. Pain management in emergency departments: A review of present protocols in The Netherlands. Eur J Emerg Med 2010;17:286-9.

28. Motov SM, Marshall JP. Acute pain management curriculum for emergency medicine residency programs. Academic Emergency Medicine 2011;18:S87-S91. 